\title{
Fault detection in a three-tank hydraulic system using unknown input observer and extended Kalman filter
}

\author{
Ayman E. O. HASSAN*, Tasnim A. A. MOHAMMED and Aşkın DEMİRKOL \\ Department of Electrical and Electronics Engineering, Sakarya University, Sakarya, Turkey \\ * Corresponding Author: ayman.hassan@ogr.sakarya.edu.tr
}

$\begin{array}{ll}\text { Submitted } & : 05 / 11 / 2019 \\ \text { Revised } & : 07 / 03 / 2021 \\ \text { Accepted } & : 21 / 03 / 2021\end{array}$

\begin{abstract}
This paper presents the problem of fault diagnosis in a three-tank hydraulic system. A mathematical model of the system is developed in order to apply two different observing algorithms. Unknown Input Observer (UIO) and Extended Kalman Filter (EKF) have been used to detect and isolate actuator and sensor faults. For Unknown Input Observer (UIO), residuals are calculated from the measured and estimated output according to the eigenvalues of the system after processed by Linear Matrix Inequality (LMI). Extended Kalman filter uses process and measurement noise variances for state estimation. Unknown Input Observer and Extended Kalman Filter's performance in fault estimation and isolation is evaluated under different scenarios. Using Extended Kalman Filter (EKF), faults can be diagnosed effectively in the presence of noise, while Unknown Input Observer (UIO) is working better in the absence of noise, and simulation results illustrate that clearly.
\end{abstract}

Keywords: Extended kalman filter; Fault detection; Fault isolation; Hydraulic system; Unknown input observer.

\section{INTRODUCTION}

The system is a combination of components that work together harmonically to accomplish a particular purpose. Error is a system event that obstructs the system from achieving its nominal condition. Fault origins as a result of the error. Fault existence interrupts the system parameters and causes its aberration, such as an obstructing actuator or sensor detriment. So, fault can be classified as plant, sensor, and actuator fault. The error can affect the interaction between the plant's components and change the communication between the system and controller. These errors alter the performance of the system by degrading the dynamical input/output (I/O) characteristics of the plant from the desired ones and hence cause system failure (Kiss et al., 2014). An error can be multiplicative or/and additive error during the system operation due to system improper functioning or instrument aging.

The literature review includes many types of research on different tank models, observer's design, and Kalman prototype, which have been used extensively in FDI.

A model of a standard three-tanks system in Chalupa et al. (2012) and Farias et al. (2018) has been tested under a design of state observer layout for states of a single or paired measurements. The presented method is robust and 
uncomplicated to prove the particularity of observers (M. Hou, 2002). The same model was used to examine nonlinear and linear multivariable feedback control to design the Fault Detection and Isolation (FDI) and Fault Tolerance Control (FTC) system (Hassan Noura, Didier Theilliol, 2009).

A decoupled linear observer has been designed to diagnose sensor, component, and actuator faults (valve, pipe, and pump) around an equilibrium point without giving any information about the fault magnitude (D. Koenig, 1997). Based on the nonlinear model of degree $\mathrm{N}$, an observer has been designed and applied to an experimental hydraulic system to detect the fault in the case of bilinear and polynomial nonlinearities by Shields \& Du (2000). In L. El Bahir \& M. Kinnaert (1998), time-varying innovation generators integrated with generalized likelihood ratio tests have been developed based on a bilinear model for fault diagnosis purposes. In Rincon-Pasaye et al. (2008), a comparison has been held between two observer types to test their reliability in fault detection using a small number of measurements from the plant. The proposed methods have been evaluated at an actual time on a model of three tanks. Residuals have been calculated successfully using nonlinear filters by Join et al. (2005). Multiple observers have been synthesized in Abdelkader. et al. (2003) to calculate the state variables of a nonlinear system which consists of a multiple sub-model with some unknown inputs. The calculation of the system's overall gain has been simplified to be the solution to the local observer's gain matrix. Faults in a multi-models system have been detected and isolated using an adaptive filter, which builds up from a group of decoupled observers (Rodrigues et al., 2008). In Koscielny (1999), a Fault diagnosis process has been accomplished using fuzzy logic relying on its ability to analyze multivalue residuals.

Wang et al. (1975) is the first researcher who has used the UIO in a system with specific unknown inputs (Hassan Noura, Didier Theilliol, 2009). The UIO has been used widely in faults detection and isolation for industrial processes and installations (Anzurez-Marin et al., 2008). UIO has been used for fault determination in a simple hydraulic plant of two tanks, and a more complicated system has been used for a system with five tanks (Tahraoui et al., 2015). while in Gaeid \& Ping (2010), it has been used to detect the fault which occurs in an induction motor.

The Extended Kalman Filter (EKF) and its various enhancement have been applied in control engineering. Therefore the process of sensor fault detection has been realized using EKF by Van Eykeren et al. (2012) also actuator fault detected by Miron et al. (2017) and Skach \& Puncochar (2017).

A new method combining an adaptive neuro-fuzzy inference system (ANFIS) and Gustafson-Kessel (GK) clustering algorithm has been used for fault identification, proper classification, and location. The GK fuzzy clustering algorithm decides the precise configuration and its parameter using the orthogonal least square (Abdullah \& Hidaka, 2018).

In this paper, we used UIO and EKF to detect the faults in the presence and absence of disturbance for a threetank hydraulic system. Then, their fault detection and isolation efficiency have been tested and compared. 


\section{HYDRAULIC MODEL OF A THREE TANKS SYSTEM}

The three-tank system shown in Fig.1 considered as our application of the FDI process.

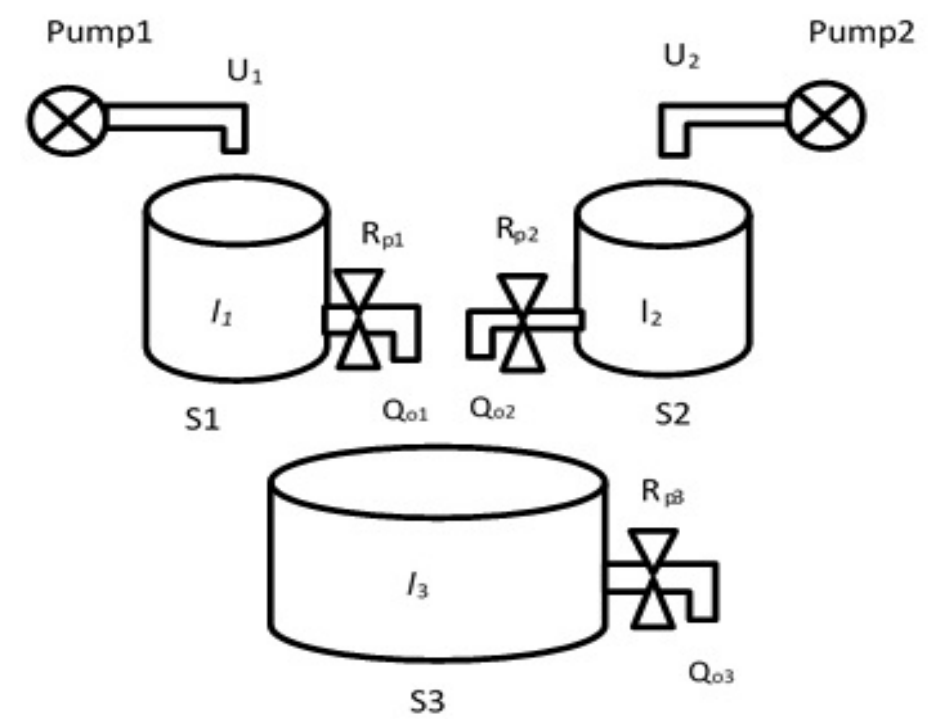

Figure 1. Hydraulic model of three tanks system.

The system in Fig. 1 is built up from two upper cylindrical tanks with the same cross-section area $\left(\mathrm{S}_{1}, \mathrm{~S}_{2}\right)$ and third lower tank, with a diverse cross-section area $S_{3}$. Pipes with resistances $\left(\mathrm{Rp}_{1}, \mathrm{Rp}_{2}\right.$, and $\left.\mathrm{Rp}_{3}\right)$ are used to connect the tanks. Pump ${ }_{1}$ supplies tank $k_{1}$ with input $U_{1}$ and pump supplies tank $_{2}$ with input $U_{2}$, while the outputs of the upper tanks $\mathrm{Q}_{01}$ and $\mathrm{Q}_{\mathrm{o} 2}$ form an input to the third tank. The outcome of the system is $\mathrm{Q}_{03}$. The resistance of the pipe can be calculated using the liquid level and tanks output, as follows:

$$
R_{p i}=\frac{\Delta l_{i j}}{Q_{o i}}
$$

while the rate of the volume change in a tank is

$$
\begin{aligned}
& \frac{d V}{d t}=\text { input }(t)-\text { output }(t), \quad \text { Where } \quad V=l \times S \\
& S \frac{d l}{d t}=\operatorname{input}(t)-\text { output }(t)
\end{aligned}
$$

By ignoring the pipeline loss, the system will be modeled using the following equations:

$$
\left\{\begin{array}{c}
S_{1} \frac{d l_{1}}{d t}=U_{1}-Q_{o 1} \\
S_{2} \frac{d l_{2}}{d t}=U_{2}-Q_{o 2} \\
S_{3} \frac{d l_{3}}{d t}=Q_{01}+Q_{02}-Q_{o 3}
\end{array}\right.
$$




$$
\begin{aligned}
& \left\{\begin{array}{c}
\frac{d Q_{01}}{d t}=\frac{-Q_{o 1}+U_{1}}{S_{1} R_{p 1}} \\
\frac{d Q_{02}}{d t}=\frac{-Q_{o 2}+U_{2}}{S_{2} R_{p 2}} \\
\frac{d Q_{o 3}}{d t}=\frac{Q_{01}+Q_{02}-Q_{o 3}}{S_{3} R_{p 3}}
\end{array}\right. \\
& \text { where }\left\{\begin{array}{c}
Q_{o j}=a_{o j} \sqrt{2 g l_{j}(t)} \\
a_{o j}=\mu_{j} S_{n}
\end{array}\right. \\
& {\left[\begin{array}{l}
\dot{l}_{1}(t) \\
\dot{l}_{2}(t) \\
\dot{l}_{3}(t)
\end{array}\right]=\left[\begin{array}{ccc}
\frac{-a_{O 1} \sqrt{2 g}}{s_{1} l_{1}} & 0 & 0 \\
0 & \frac{-a_{o 2} \sqrt{2 g}}{s_{2} l_{2}} & 0 \\
\frac{a_{o 1} \sqrt{2 g}}{s_{3} l_{1}} & \frac{a_{O 2 \sqrt{2 g}}}{s_{3} l_{2}} & \frac{-a_{O 3} \sqrt{2 g}}{S_{3} l_{3}}
\end{array}\right]\left[\begin{array}{l}
l_{1} \\
l_{2} \\
l_{3}
\end{array}\right]+\left[\begin{array}{cc}
\frac{1}{S_{1}} & 0 \\
0 & \frac{1}{s_{2}} \\
0 & 0
\end{array}\right]\left[\begin{array}{l}
U_{1} \\
U_{2}
\end{array}\right]} \\
& {\left[\begin{array}{l}
\dot{Q}_{o 1} \\
\dot{Q}_{o 2} \\
\dot{Q}_{o 3}
\end{array}\right]=\left[\begin{array}{ccc}
\frac{-1}{S_{1} R_{p 1}} & 0 & 0 \\
0 & \frac{-1}{S_{2} R_{p 2}} & 0 \\
\frac{1}{S_{3} R_{p 3}} & \frac{1}{S_{3} R_{p 3}} & \frac{-1}{S_{3} R_{p 3}}
\end{array}\right]\left[\begin{array}{l}
Q_{o 1} \\
Q_{o 2} \\
Q_{o 3}
\end{array}\right]+\left[\begin{array}{cc}
\frac{1}{S_{1} R_{p 1}} & 0 \\
0 & \frac{1}{S_{2} R_{p 2}} \\
0 & 0
\end{array}\right]\left[\begin{array}{l}
U_{1} \\
U_{2}
\end{array}\right]}
\end{aligned}
$$

The model represented by equations ( 7 and 8 ) is a nonlinear model; after discretization by Euler method with sampling time $t=0.1_{S}$, and linearized with $x_{e 1}, x_{e 2}$, and $x_{e 3}$ as an equilibrium point given (Witczak et al., 2016a) :

$$
\begin{gathered}
x_{1}=l_{1}, x_{2}=l_{2}, x_{3}=l_{3} \\
A_{e}=\left[\begin{array}{ccc}
\frac{-\left(a_{o 1}\right)^{2}(g)}{S_{1} U_{e 1}} & 0 & 0 \\
0 & \frac{-\left(a_{o 2}\right)^{2}(g)}{S_{2} U_{e 2}} & 0 \\
\frac{\left(a_{o 1}\right)^{2}(g)}{S_{3} U_{e 1}} & \frac{\left(a_{o 1}\right)^{2}(g)}{S_{3} U_{e 2}} & \frac{-\left(a_{o 1}\right)^{2}(g)}{S_{3}\left(U_{e 1}+U_{e 2}\right)}
\end{array}\right] \\
B_{e}=\left[\begin{array}{cc}
\frac{1}{S_{1}} & 0 \\
0 & \frac{1}{S_{1}} \\
0 & 0
\end{array}\right], C_{e}=\left[\begin{array}{lll}
1 & 0 & 0 \\
0 & 1 & 0 \\
0 & 0 & 1
\end{array}\right] \text { and } D_{e}=0
\end{gathered}
$$

\section{THEORY}

\section{Unknown Input Observer (UIO)}

Signal residual is the difference between the actual output and estimated output. By observing it, the process of fault detection can be achieved according to the following equation:

$$
r(k)=y(k)-C \hat{x}(k)
$$


Equation (14) describes a nonlinear system with unknown components, which can be assumed as an additive term. This system model is used for observer design.

$$
\left\{\begin{array}{c}
x(k+1)=A x(k)+B u(k)+\mathrm{F}_{x} f(k)+E d(k)+\mathrm{V}(\mathrm{x}(k), \mathrm{u}(k)) \\
y(t)=C x(k)+\mathrm{F}_{y} f(k)
\end{array}\right.
$$

where $x \in h^{m}$ is the state vector, $u \in h_{l}^{n}$ is the control input vector, $y \in h_{l}^{n}$ is the output vector, and $d(t) \in h^{q}$ is the vector of unknown input. $A, B, C$, and $E$ are matrices of proper sizes.

The UIO design is created based on the system equation (14). Without considering the system disturbance and unknown inputs, the estimation error vector $e(t)$ tends to zero. Full order observer characterized as follows:

$$
\left\{\begin{array}{c}
\dot{\xi}(k+1)=F \xi(k)+T B u(k)+K y(k)+T V\left(\hat{x}_{k}, u_{k}\right) \\
\hat{x}(k)=\xi(k)+H y(k)
\end{array}\right.
$$

where $\hat{x}(k) \in \Re^{m}$ is the estimated state vector, $\xi(k) \in \Re^{n}$ is the state vector of full order observer. The values of matrix $H, K, T$, and $F$ are calculated using Linear Matrix Inequality (LMI) to satisfy the uncoupling of unknown input and consider the design requirement. (Anzurez-Marin et al., 2008; Witczak et al., 2016b). $e(t)$ tends to have zero value if the matrix $\mathrm{F}$ is Hurwitz $\hat{x}(k) \rightarrow x(k)$, and the following constraints are satisfied:

$$
\left\{\begin{array}{c}
(I+H C) E=0 \\
T=I+H C \\
F=A-H C A-K_{1} C \\
K_{2}=F H
\end{array}\right.
$$

where $\mathrm{K}=\mathrm{K}_{1}+\mathrm{K}_{2}$

The observer (15) must fulfill the following necessary and sufficient constraints to be UIO for the system in equation (14):

- $\quad(g(a, u)-g(b, u))^{T}(a-b) \leq(a-b)^{T} M(a-b), \quad \forall a, b \in X \mathbb{X}, u \in \mathbb{U}$

- $\quad(g(a, u)-g(b, u))^{T}(g(a, u)-g(b, u)) \leq(a-b)^{T} M^{T} M(a-b), \quad \forall a, b \in X \mathbb{X}, u \in \mathbb{U}$

- $\quad \operatorname{Rank}(C E)=\operatorname{rank}(E)$.

- $\quad$ The detectability of pair $(C, A 1)$.

Where

$$
\left\{\begin{array}{c}
A_{1}=A-E\left[(C E)^{T} C E\right]^{-1}(C E)^{T} C A=T A \\
H=E\left[(C E)^{T} C E\right]^{-1}(C E)^{T}
\end{array}\right.
$$

Here we observe that the number of independent measurements must be less than the maximum number of uncoupled disturbances. After satisfied the detectability condition of the pair $\left(C, A_{I}\right)$, then the proper value of $K_{I}$ should be selected to guarantee the stability of $F=A_{1}-K_{1} C$, which is an essential step. 


\section{Extended Kalman Filter (EKF)}

Kalman Filter is an ideal estimator designed to deal with model-based linear systems. The Extended Kalman Filter algorithm is an advanced version of the ordinary Kalman filter that works with nonlinear model-based systems. The following equations represent a general form of Extended Kalman Filter for a nonlinear discrete-time system. State-space equation:

$$
x(k+1)=f[x(k), u(k)]+G w(k)
$$

Measurement equation:

$$
y(k)=g[x(k), u(k)]+H w(k)+v(k)
$$

While $x$ is the state vector of $\mathrm{n}$ state variables, $u$ is the input vector of $\mathrm{m}$ input variables. $F$ is the system vector function, and $w$ is a random (white) disturbance (or process noise) vector with auto-covariance $Q$. $G$ is the process noise gain matrix relating the process noise to the state variables.

$Y$ is the measurement vector of $r$ measurement variables, $g$ is the measurement vector function. $H$ is a gain matrix relating the disturbances directly to the measurements, and $v$ is a random (white) measurement noise vector with auto-covariance $R$.

State estimation process using Kalman Filter can be summarized as follows:

- Set the initial value of the predicted state.

$$
x_{p}(0)=x_{\text {init }}
$$

- Using the predicted state estimate, calculate the expected measurement estimate.

$$
y_{p}(k)=g\left[x_{p}(k)\right]
$$

- The measurement estimate error is calculated by subtracting the measurement $y(k)$ from the predicted measurement $y_{p}(k)$.

$$
e(k)=y(k)-y_{p}(k)
$$

- $\quad$ Find the corrected state estimate $x_{c}(k)$ by adding the corrective term $K e(k)$ to the predicted state estimate $x_{p}(k)$.

$$
x_{c}(k)=x_{p}(k)+K e(k)
$$

- $\quad$ Find the predicted state estimate for the next time step., $x_{p}(k+1)$, using the present state estimate $x_{c}(k)$ and the known input $u(k)$ in process model:

$$
x_{p}(k+1)=f\left[x_{c}(k), u(k)\right]
$$




\section{METHODOLOGY}

\section{Fault Detection in Three-Tank Model Using UIO}

The following parameters have been applied to the model:

$$
\begin{gathered}
S_{1}=0.0154 \mathrm{~m}^{2}, \quad S_{2}=S_{1} \text { and } S_{3}=3 \times S_{1} \\
S_{n}=6 \times 10^{-5} \mathrm{~m}^{2}, \quad g=9.81 \frac{\mathrm{m}}{\mathrm{s}^{2}}, \quad \mu=0.6, \\
U_{e 1}=3 \times 10^{-5} \mathrm{~m}^{3} / \mathrm{s}, \quad U_{e 2}=5 \times 10^{-5} \mathrm{~m}^{3} / \mathrm{s}
\end{gathered}
$$

$S_{n}$ : the pipe section area, $g$ : Acceleration of gravity, and $\mu_{i}$ : flow coefficient of the tank $i$.

These parameter values were taken from several previous studies (Tahraoui et al., 2015) (Hassan Noura, Didier Theilliol, 2009), and after applying transformation matrix and LMI solver, the following matrix has been achieved (Buciakowski et al., 2017; Zhang et al., 2016):

$$
\begin{aligned}
& {\left[\begin{array}{l}
\dot{x}_{1} \\
\dot{x}_{2} \\
\dot{x}_{3}
\end{array}\right]=\left[\begin{array}{ccc}
-5.504 & 0 & 0 \\
0 & -4.128 & 0 \\
-1.835 & 1.376 & -0.786
\end{array}\right]\left[\begin{array}{l}
x_{1} \\
x_{2} \\
x_{3}
\end{array}\right]+\left[\begin{array}{cc}
64.935 & 0 \\
0 & 64.935 \\
0 & 0
\end{array}\right]\left[\begin{array}{l}
U_{1} \\
U_{2}
\end{array}\right]+\left[\begin{array}{l}
1 \\
1 \\
0
\end{array}\right] f_{a}(t)+\left[\begin{array}{l}
1 \\
1 \\
0
\end{array}\right] d(t)+} \\
& {\left[\begin{array}{l}
1 \\
1 \\
0
\end{array}\right] V(x(k), u(k))}
\end{aligned}
$$

$$
y=\left[\begin{array}{lll}
1 & 0 & 0 \\
0 & 1 & 0 \\
0 & 0 & 1
\end{array}\right]\left[\begin{array}{l}
x_{1} \\
x_{2} \\
x_{3}
\end{array}\right]+\left[\begin{array}{l}
0 \\
0 \\
1
\end{array}\right] f_{s}(t)+\left[\begin{array}{l}
0 \\
0 \\
1
\end{array}\right] d(t)
$$

Observer matrix has been obtained from LMI solver:

$$
\left\{\begin{array}{c}
F=\left[\begin{array}{ccc}
-0.79 & 0 & 0 \\
0 & -3.25 & 0 \\
0 & 0 & -0.50
\end{array}\right], H=\left[\begin{array}{cc}
0 & 0 \\
0.50 & 0 \\
0 & 1
\end{array}\right] \\
k=\left[\begin{array}{cc}
1.83 & 0 \\
-0.50 & 0 \\
0 & -0.50
\end{array}\right] \text { and } T=\left[\begin{array}{ccc}
1 & 0 & 0 \\
0 & 0.5 & 0 \\
0 & 0 & 0
\end{array}\right]
\end{array}\right.
$$

\section{Fault Detection in Three-Tank Model Using EKF}

By assuming that the change in outflow rate is approximately zero,

$$
x_{1}=Q_{o 1}, x_{2}=Q_{o 2}, \text { and } x_{3}=Q_{o 3}
$$




$$
\left[\begin{array}{l}
\dot{x}_{1} \\
\dot{x}_{2} \\
\dot{x}_{3}
\end{array}\right]=\left[\begin{array}{ccc}
\frac{-1}{s_{1} R_{p 1}} & 0 & 0 \\
0 & \frac{-1}{s_{2} R_{p 2}} & 0 \\
\frac{1}{s_{3} R_{p 3}} & \frac{1}{S_{3} R_{p 3}} & \frac{-1}{s_{3} R_{p 3}}
\end{array}\right]\left[\begin{array}{l}
x_{1} \\
x_{2} \\
x_{3}
\end{array}\right]
$$

The state jacobian:

$$
J_{S}=\left[\begin{array}{ccc}
\frac{-T_{S}}{S_{1} R_{p 1}} & 0 & 0 \\
0 & \frac{-T_{S}}{S_{2} R_{p 2}} & 0 \\
\frac{T_{S}}{S_{3} R_{p 3}} & \frac{T_{S}}{S_{3} R_{p 3}} & \frac{-T_{S}}{S_{3} R_{p 3}}
\end{array}\right]
$$

The measurement jacobian:

$$
J_{m}=\left[\begin{array}{lll}
1 & 0 & 0 \\
0 & 1 & 0 \\
0 & 0 & 1
\end{array}\right]
$$

The process noise covariance:

$$
N_{Q}=\left[\begin{array}{ccc}
0.01 & 0 & 0 \\
0 & 0.01 & 0 \\
0 & 0 & 0.01
\end{array}\right]
$$

The measurement noise covariance:

$$
N_{m}=\left[\begin{array}{ccc}
10^{-4} & 0 & 0 \\
0 & 10^{-4} & 0 \\
0 & 0 & 10^{-4}
\end{array}\right]
$$

\section{RESULTS}

The results achieved below using MATLAB R2020b installed on Casper Excalibur with processor Intel ${ }^{\circledR}$ core тM i7-10750H CPU @ 2.60 GHz and 32 GB RAM.

\section{Results of Fault Detection using Unknown Input Observer (UIO)}

The error which occurs in the system as a result of the unknown input can be presented in simulation in several scenarios; actuator fault (first input ) applied when $4 \leq t \leq 9 \mathrm{~s}$, second actuator fault (second input ) applied when $13 \leq$ $t \leq 17 \mathrm{~s}$, and output sensor fault when $26 \leq t \leq 34 \mathrm{~s}$. The proposed system has two measured inputs (pump1 and pump2) and one measured output (sensor). Fig. 2 shows the observers' response in the absence of fault and noise for UIO. 

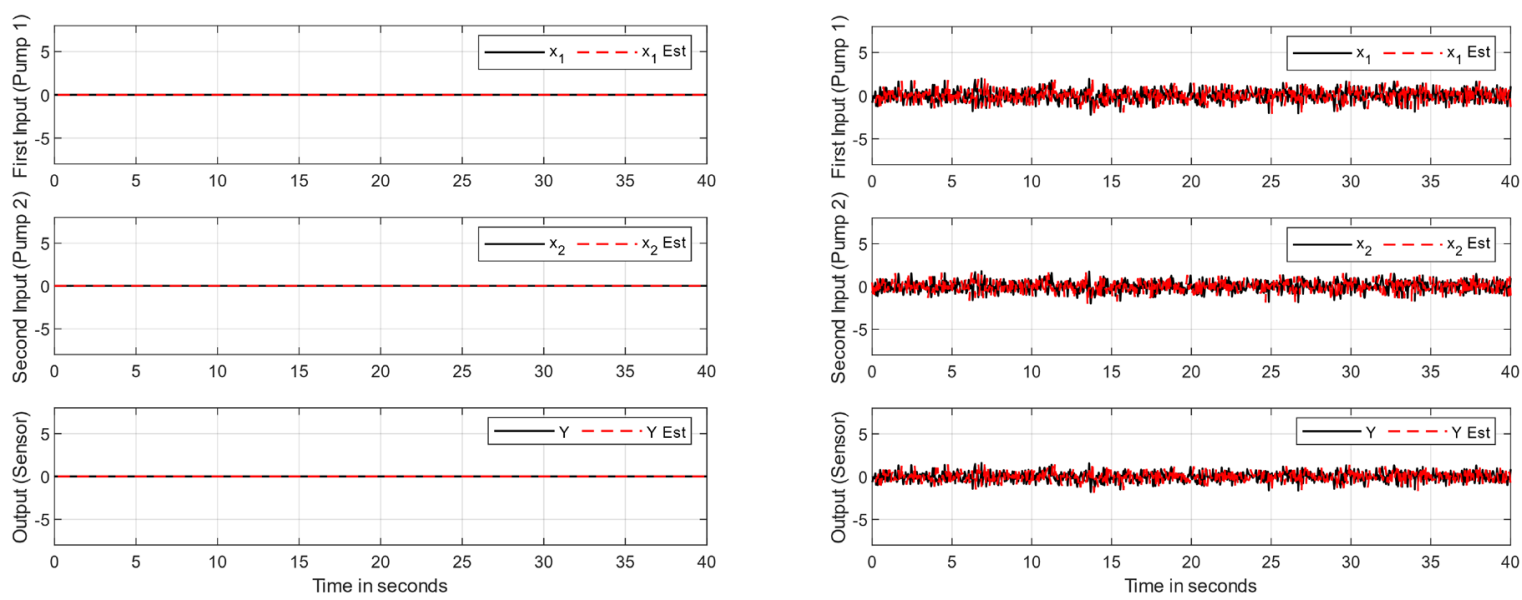

(a)

(b)

Figure 2. System without Fault (a) UIO without noise (b) UIO with noise.
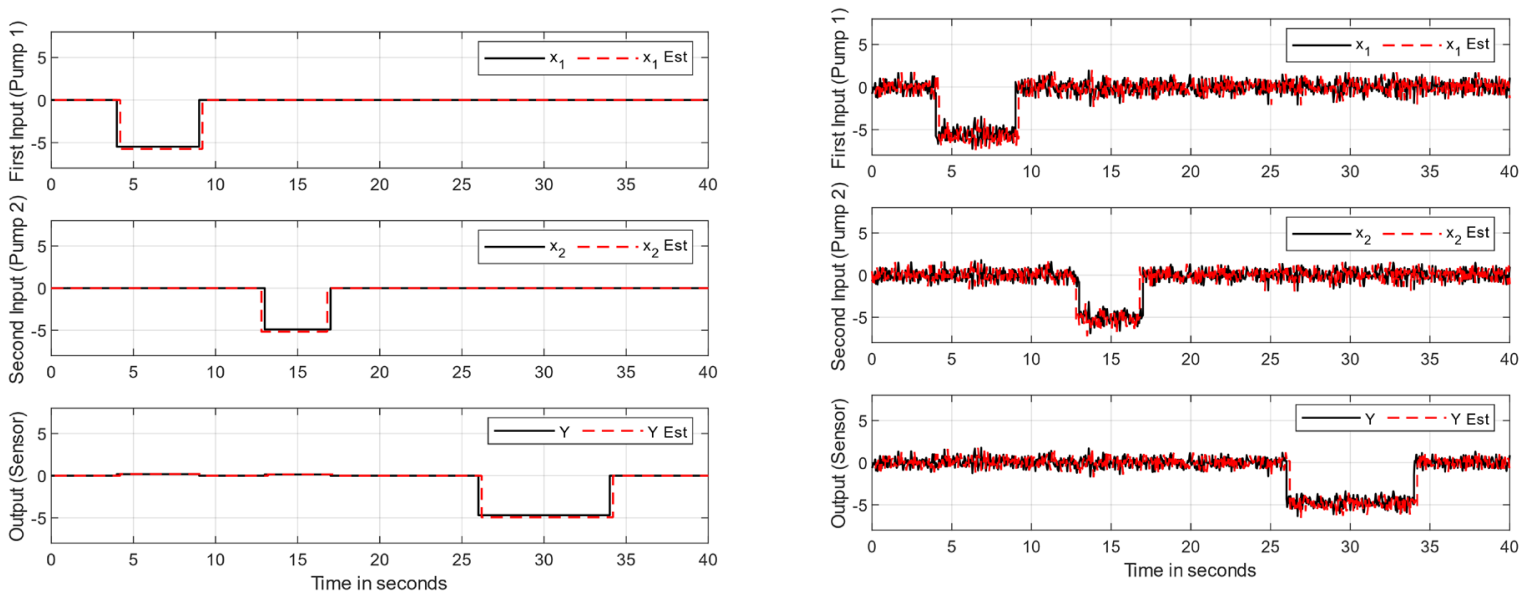

(a)

(b)

Figure 3. System with fault (a) Faulty UIO without noise (b) Faulty UIO with noise.

From Fig. 3, faults appear in the two inputs and sensor output in the absence of noise for UIO, while the noise exists in the case of EKF.

\section{Results of Fault Detection using Extended Kalman Filter (EKF)}

To evaluate the ability of the EKF to estimate actuator and sensor fault two scenarios have been implemented: the presence of noise with and without fault. Because without the process noise, the EKF model convergence becomes a difficult issue. 


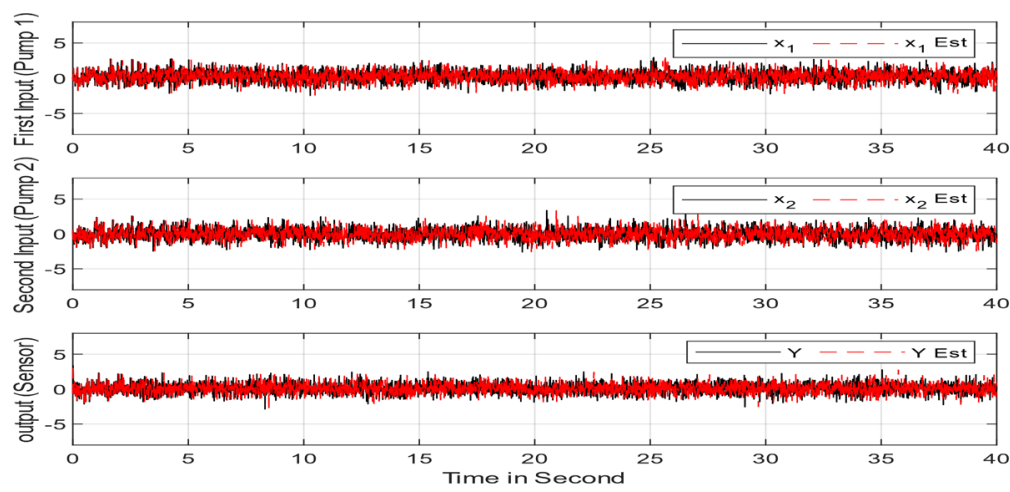

Figure 4. Extended Kalman Filter response without fault.

Fig.10 shows the response of the EKF in the case of the nonexistence of fault.

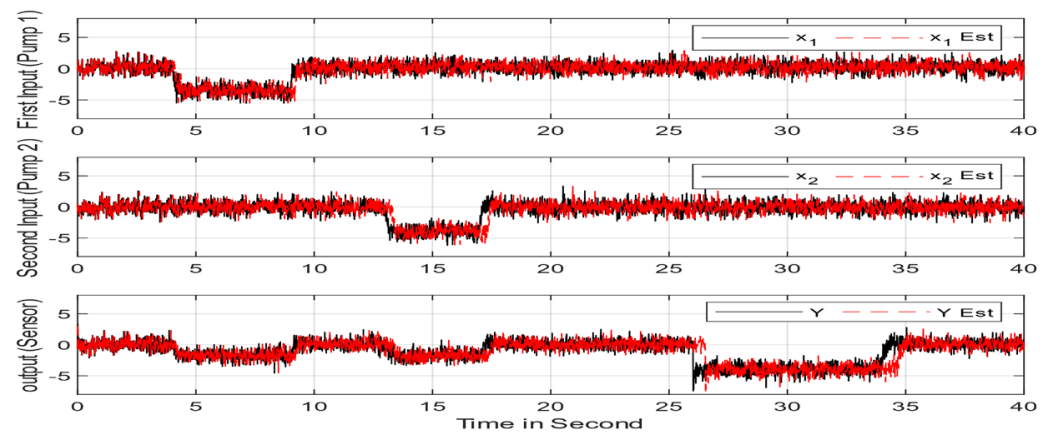

Figure 5. Extenend Kalman filter response with fault.

Fig. 5 shows the response of EKF in case of a complete model fault.

Table 1. Statistical analysis of Unknown Input Observer.

\begin{tabular}{|c|c|c|c|c|}
\hline \multicolumn{4}{|c|}{ Unknown Input Observer } \\
\hline \multirow{2}{*}{ case } & \multicolumn{2}{|c|}{ Without Noise } & \multicolumn{2}{c|}{ With Noise } \\
\cline { 2 - 5 } & without fault & With Fault & without fault & With Fault \\
\hline RMSEX1 & 0.0017 & 1.74 & 0.71 & 1.89 \\
\hline RMSEX2 & 0.0026 & 1.40 & 0.64 & 1.56 \\
\hline RMSEY & 0.0001 & 1.88 & 0.57 & 1.95 \\
\hline
\end{tabular}


Table 2. Statistical analysis of Extended Kalman Filter.

\begin{tabular}{|c|c|c|}
\hline \multirow{2}{*}{ case } & \multicolumn{2}{|c|}{ With Noise } \\
\cline { 2 - 3 } & without fault & With Fault \\
\hline RMSEX1 & 0.40 & 0.88 \\
\hline RMSEX2 & 0.41 & 0.88 \\
\hline RMSEY & 0.75 & 1.44 \\
\hline
\end{tabular}

Table 1 and Table 2 show the two observers' statistical analysis, which indicates the Extended Kalman filter's efficiency in cases of noise. In contrast, Unknown Input Observer is strongly recommended for the absence of noise.

\section{CONCLUSIONS}

In this paper, Unknown Input Observer (UIO) and Extended Kalman Filter (EKF) were applied to a fault diagnosis problem. A nonlinear model of three-tank hydraulic system is used as an application; the fault diagnosis algorithms were tested and simulated in Matlab. According to the simulation results, the observer may look to be less complicated than the Extended Kalman Filter. On the other hand, the Extended Kalman filter can solve the potential danger, which appears when using the UIO.

The state estimation process using UIO might be too noisy due to the unavoidable measurement noise, because, in the sense of the least mean square, it does not compute any optimal state, which is the main problem. Fast dynamics systems require a large gain estimator $(\mathrm{K})$, which causes noise measured in the output that significantly affects the state estimates.

The state estimation process using Extended Kalman Filter is better, because it uses the process and measurement noise variances, which is more accessible than using eigenvalues of the error-model.

\section{REFERENCES}

Abdelkader., A., Mohammed., C., Didier., M., \& Jos6., R. (2003). State estimation via multiple observer. The three tank system. 5th IFAC Symposium on Fault Detection, Supervision and Safety for Technical Processes, $1227-1232$.

Abdullah, A., \& Hidaka, K. (2018). Fault identification usingcombined adaptive neuro-fuzzy inference system and Gustafson-Kessel algorithm. In Journal of Engineering Research (Vol. 6, Issue 1). https://kuwaitjournals.org/jer/index.php/JER/article/view/3241

Anzurez-Marin, J., Pitalúa-Díaz, N., Cuevas-Silva, O., \& Villar-García, J. (2008). Unknown inputs observers design for fault detection in a two-tank hydraulic system. Proceedings - Electronics, Robotics and Automotive Mechanics Conference, CERMA 2008, 373-378. https://doi.org/10.1109/CERMA.2008.76 
Buciakowski, M., Witczak, M., Puig, V., Rotondo, D., Nejjari, F., \& Korbicz, J. (2017). A bounded-error approach to simultaneous state and actuator fault estimation for a class of nonlinear systems. Journal of Process Control, 52, 14-25. https://doi.org/10.1016/j.jprocont.2017.01.002

Chalupa, P., Novák, J., \& Bobál, V. (2012). Comprehensive model of DTS200 three tank system in Simulink. International Journal of Mathematical Models and Methods in Applied Sciences, 6(2), 358-365.

D. Koenig, S. N. and T. C. (1997). An original approach for actuator and component fault detection and isolation. IFAC Proceedings Volumes, 30(18), 97-107. https://doi.org/10.1016/S1474-6670(17)42387-1

Farias, A. O., Queiroz, G. A. C., Bessa, I. V., Medeiros, R. L. P., Cordeiro, L. C., \& Palhares, R. M. (2018). Sim3Tanks: A Benchmark Model Simulator for Process Control and Monitoring. IEEE Access, 6(October), 62234-62254. https://doi.org/10.1109/ACCESS.2018.2874752

Gaeid, K. S., \& Ping, H. W. (2010). Induction motor fault detection and isolation through unknown input observer. Scientific Research and Essays, 5(20), 3152-3159.

Hassan Noura, Didier Theilliol, J.-C. P. and A. C. (2009). Application to a Three-tank System. In Fault-tolerant Control Systems Design and Practical Applications (Issue Mm, pp. 109-156).

Join, C., Ponsart, J.-C., Sauter, D., \& Theilliol, D. (2005). Nonlinear filter design for fault diagnosis: application to the three-tank system. IEE Proceedings - Control Theory and Applications, 55-64.

Kiss, A. M. N., Marx, B., Mourot, G., \& Ragot, G. S. and J. (2014). State estimation of the three-tank system using a multiple model. 48th IEEE Conference on Decision and Control and 28th Chinese Control Conference, $7795-7800$.

Koscielny, J. M. (1999). Application of fuzzy logic for fault isolation in a three-tank system. 14th World Congress of IFAC, 32(2), 7754-7759.

L. El Bahir, \& M. Kinnaert. (1998). Fault detection and isolation for a three-tank system based on a bilinear model of the supervised process. United Kingdom Automatic Control Council International Conference on Control, 2, 1486 1491. https://doi.org/10.1109/acc.2006.1657600

M. Hou, Y. S. X. and R. J. P. (2002). Observer design for a three-tank system. IFAC Proceedings Volumes, 35(1), 327-332. https://doi.org/10.3182/20020721-6-ES-1901.00216

Miron, M., Frangu, L., \& Caraman, S. (2017). Actuator fault detection using extended Kalman filter for a wastewater treatment process. 2017 21st International Conference on System Theory, Control and Computing (ICSTCC), 583-588.

Rincon-Pasaye, J. J., Martinez-Guerra, R., \& Soria-Lopez, A. (2008). Fault diagnosis in nonlinear systems: An application to a three-tank system. Proceedings of the American Control Conference, 152(1), 2136-2141. https://doi.org/10.1109/ACC.2008.4586808

Rodrigues, M., Theilliol, D., Medina, M. A., \& Sauter, D. (2008). A fault detection and isolation scheme for industrial systems based on multiple operating models. Control Engineering Practice, 30(18), 225-239. https://doi.org/10.1016/s1474-6670(17)42536-5

Shields, D. N., \& Du, S. (2000). An assessment of fault detection methods for a benchmark system. IFAC Proceedings Volumes, 33(11), 915-920. https://doi.org/10.1016/S1474-6670(17)37478-5

Skach, J., \& Puncochar, I. (2017). Input Design for Fault Detection Using Extended Kalman Filter and $\begin{array}{llll}\text { Reinforcement } \quad \text { Learning. } & \text { 7FAC-PapersOnLine, }\end{array}$ https://doi.org/10.1016/j.ifacol.2017.08.1461

Tahraoui, S., Meghebbar, A., Boubekeur, D., \& Boumediene, A. (2015). Fault detection in a five tank hydraulic system. EEA - Electrotehnica, Electronica, Automatica, 63(3), 51-59. 
Van Eykeren, L., Chu, Q. P., \& Mulder, J. A. (2012). Sensor fault detection and isolation using adaptive extended Kalman filter. IFAC Proceedings Volumes, 45(20), 1155-1160.

Witczak, M., Buciakowski, M., Puig, V., Rotondo, D., \& Nejjari, F. (2016a). An LMI approach to robust fault estimation for a class of nonlinear systems. INTERNATIONAL JOURNAL OF ROBUST AND NONLINEAR CONTROL Int. J. Robust Nonlinear Control, 26(7), 1530-1548. https://doi.org/10.1002/rnc.3365

Witczak, M., Buciakowski, M., Puig, V., Rotondo, D., \& Nejjari, F. (2016b). An LMI approach to robust fault estimation for a class of nonlinear systems. International Journal of Robust and Nonlinear Control, 26(7), 1530-1548. https://doi.org/10.1002/rnc.3365

Zhang, J., Swain, A. K., \& Nguang, S. K. (2016). Robust Observer-Based Fault Diagnosis for Nonlinear Systems Using $M A T L A B \circledR$. Springer International Publishing. https://doi.org/10.1007/978-3-319-32324-4 